\title{
EDITORIAL
}

\section{Editorial: Post-publication Discussion}

\author{
WCW Chu \\ Editor-in-Chief, Hong Kong Journal of Radiology
}

Providing a way for readers to comment on and discuss published articles is a fundamental role of academic journals. ${ }^{1}$ Traditionally, such comments were restricted to Letters to the Editor and published in the pages of the journal. More recently, as journals have expanded their online presence, so too commentary has evolved. Some journals now permit comments on articles directly on the website, whereas others have embraced thirdparty websites such as PubPeer (https://pubpeer.com/) to provide a platform for comments. Wherever they are published, these comments serve to question or corroborate results or conclusions. In this way, they ensure the integrity and accuracy of published works and, in some cases, may lead to correction of the published literature. In order to facilitate post-publication discussion, Hong Kong Journal of Radiology (HKJR) accepts Letters to the Editor and Brief Communications.

Letters to the Editor comment on, question, support, or criticise a topic of current interest, typically referring to an article published in a recent issue of the journal. They should be concise and to the point (typically 100200 words), with few references ( $\leq 5)$. No introduction or discussion is needed, and figures or tables are permitted only exceptionally. The Letter should begin by specifying the particular point of focus, which is usually a result or conclusion from a recent article. The author's opinion is then presented respectfully and constructively. If referring to a recent article, the Letter is usually sent to the authors of that article, who are then given the opportunity to provide a response. Any response is published together with the Letter. Letters are reviewed by the Editorial Board to assess their relevance and importance to HKJR and our readers.
Brief Communications are short articles (500-1500 words) that can fulfil a variety of roles. In the context of post-publication discussion, Brief Communications provide a longer format than Letters, allowing for broader or more in-depth analysis. In order to stand alone, a short introduction must be included, providing some background and a clear statement of purpose. The authors' perspective on the original article should be clearly presented, for example any perceived valuable contributions to the field, controversial results, or interesting lessons. The authors' opinion should be supported by the literature ( $\leq 15$ references) and may be supplemented with example case(s) or data from the authors' own experience. Brief Communications are peer reviewed by at least two experts to ensure the accuracy and reliability of the presented material.

The Editorial Board and I would like to invite our readers to submit their valuable comments as Letters to the Editor or Brief Communications for consideration for publication in HKJR. The views of our readers have always been very welcome, and as part of ongoing improvements to HKJR, we would like to encourage more submissions of this type. At the same time, Editorial Board members will be writing more Editorials, to summarise future issues, to highlight key papers or topics, and to put the research in perspective, to increase the value for readers. These enhancements are part of a concerted effort to expand the reach and impact of HKJR.

\section{REFERENCE}

1. Principles of Transparency and Best Practice in Scholarly Publishing. Committee on Publication Ethics; 2014. https://doi. org/10.24318/cope.2019.1.12 